hep-th/0009057

SLAC-PUB-8611

\title{
Extended Objects from Warped Compactifications of M theory
}

\author{
Eva Silverstein \\ Department of Physics and SLAC \\ Stanford University \\ Stanford, CA 94305/94309
}

\begin{abstract}
We study the massive spectrum of fully wrapped branes in warped M-theory compactifications, including regimes where these states are parametrically lighter than the Planck scale or string scale. We show that many such states behave classically as extended objects in the noncompact directions in the sense that their mass grows with their size as measured along the Poincare slices making up the noncompact dimensions. On the other hand these states can be quantized in a nontrivial regime: in particular their spectrum of excitations in a limited regime can be obtained by a warped Kaluza-Klein reduction from ten dimensions. We briefly discuss scattering processes and loop effects involving these states, and also note the possibility of an exponential growth in the number of bound states of these objects as a function of energy.
\end{abstract}

September 2000 


\section{Introduction}

One of the features of the web of M-theory duals is the presence of various limits in which a distinctive spectrum of states emerges as the lightest excitation above the (super-) gravity modes. For example in some limits strings dominate, in others particles including those coming from wrapped branes.

Compactifications of M theory down to $d$ Poincare-invariant dimensions are generically warped products, with $d$-dimensional Minkowski space varying over a compact $11-d$ manifold $K$. That is, the metric is of the form

$$
d s^{2}=a(y) d x_{\|}^{2}+H_{i j}(y) d y^{i} d y^{j}
$$

where $x_{\|}^{\mu}, \mu=0, \ldots, d-1$ are coordinates along the Poincare slices and $y_{i}, i=d, \ldots, 10$ are coordinates along the internal manifold. In this paper we will study basic aspects of the massive spectrum in such compactifications (focusing on two concrete examples: Horava-Witten theory compactified on $K 3$ [1], and type I' theory as studied by Polchinski and Witten [2] compactified on a 5-torus).

We will find objects which behave in the noncompact $x_{\|}$directions in a way that is in some sense intermediate between particles and higher branes. These come from ordinary particles or fully wrapped branes in the ten or eleven-dimensional description of the physics, but ones for which the mass and effective size as measured along the Poincare slices varies over the compactification in such a way that the energy grows as some positive power of the thickness $\delta x_{\|}$. We will refer to such objects as elastic states. By size of the object we will mean its thickness or the size of the short-distance cutoff scale, measured with respect to the $x_{\|}$coordinates, as probed in appropriate scattering experiments. As we will discuss, in some regimes this is greater than or equal to the Compton wavelength of our excitations so that in these cases this is a classically intuitive accounting of the size scale.

In a general warped geometry of the form (1.1), the size $R$ of an object as measured in the $x_{\|}$coordinates $\left(R \equiv \delta x_{\|}\right)$depends on its characteristic proper size $r_{0}(y)$ and on the warp factor $a(y)$, via the relation

$$
R=\frac{1}{\sqrt{a(y)}} r_{0}(y)
$$

The energy of an object depends on $a(y)$ and the metric $H_{i j}(y)$ of the internal space (as well as the profiles of any additional fields with nontrivial VEVs in a given background). As we 
move the object around $K$ to different values of $y_{i}$, its effective size changes and its energy changes. For ordinary point-particle quantum field theory the energy is inversely related to the size at high energies.1 We will see in our examples that the size instead grows as some positive power of the energy for certain massive states in warped compactifications. This variation in the size of the object results from a combination of its intrinsic thickness in $11 d \mathrm{M}$ theory and the warping of the metric involved in measuring its size in the $x_{\|}$ coordinates.

The phenomenon of growth of the size of states with energy is generic at high energies due to the presence of black holes, and is seen in other contexts in many corners of M theory (see [6] for a recent example and references to earlier ones). One result of our analysis is that warped compactifications provide another place where this intriguing effect emerges. Our analysis uses the basic relations arising from the gravitational redshift in geometries of the form (1.1) that also comes into the UV/IR correspondence developed for AdS space in studying the AdS/CFT correspondence [7]; our work involves in some ways a generalization of those studies to other warped geometries where there is no (evident) field theory dual, but where the energy/size relation can be studied directly in the gravity theory. The wrapped branes we study are in some sense generalizations of the baryon states studied in backgrounds with quantum field theory duals [8].

Although the elastic states have a growth in size with energy reminiscent of branes, their spectrum and interactions at long distances can be calculated via ordinary KaluzaKlein reduction in the warped geometry (1.1). This spectrum of states, while growing in number faster with energy than for ordinary unwarped Kaluza-Klein states on an circle, exhibits fewer degrees of freedom than a real brane. In this sense our elastic states are intermediate between particles and branes (and perhaps analogous to elastic solids, hence our nomenclature). However if we consider the set of potential bound states of any number $N$ of elastic states, we obtain a spectrum with enough degrees of freedom to describe modes of a continuous medium, and we discuss at the end a speculation for using these ideas to construct and quantize real branes using warped compactifications.

Direct couplings of objects of different size in this type of system are suppressed due to their separation in the internal dimensions, much like in similar systems studied

1 In AdS realizations of quantum field theory one sees membrane-shaped objects when a particle falls toward the horizon in AdS [3] 国]5], with their energy decreasing as their size increases as opposed to the cases we will focus on here. 
in the context of the AdS/CFT correspondence. The size of these objects however does manifest itself in the cross sections for scattering processes mediated by electromagnetism and gravity.

One limitation of this approach is that these objects in the regime we study them here are heavier than certain Kaluza-Klein modes of massless $11 d$ supergravity fields. So in the $d$-dimensional description, we see one or more extra dimensions before we see the elastic states. As we will see, the elastic states can be made very nearly stable in this same limit, and therefore at least do not decay into the lighter KK modes. (In some circumstances it may transpire that there is an AdS/CFT-like duality between a $d$-dimensional QFT and the supergravity modes, coupled to the rest of the system including the elastic states. Then the elastic states could be studied consistently in a completely $d$-dimensional description of the system.)

Another limitation is in our ability to calculate detailed physical quantities at the length scale corresponding to the size of the objects here. In the Horava-Witten example, the size is determined by the intrinsice thickness of the M2-brane, whose proper size is of order $l_{11}$. We can ameliorate this problem by considering the classical physics of a large- $\mathrm{N}$ collection of such objects, as we discuss in $\S 2$ and $\S 3$. To discuss the quantum behavior of systems of this sort, it might be useful to find an example in which the basic object is a (possibly wrapped) fundamental string, in a perturbative regime.

The paper is organized as follows. In $\S 2$ we will introduce the Horava-Witten example and compute the energy/size relations that arises there (explaining in particular how the size is defined and determined). We will work out the scale of masses and sizes arising from Kaluza-Klein reduction. In $\S 3$ we work out (in less detail) the same procedure for type I' theory. In $\S 4$ we will provide a preliminary discussion of several interesting aspects and applications of these results. In $\S 4.1$ we will comment on the similarities and differences with branes, and discuss a possibility for an exponentially growing spectrum of bound states. In $\S 4.2$ we discuss scattering amplitudes and loop effects in these models. $\S 4.3$ we conclude with a discussion of other issues and future directions.

\section{Horava-Witten Example}

\subsection{Mass-Size relation in $H W$ theory}

M theory compactified on $S^{1} / \mathbb{Z}_{2} \times K 3$ has a warped metric [1]

$$
d s^{2}=y^{-1 / 3} d x_{\|}^{2}+y^{2 / 3}\left(d K^{2}+\frac{V_{0}^{2}}{l_{11}^{6}} d y^{2}\right)
$$


where $l_{11}$ is the eleven-dimensional Planck length, and $y=c+2 \sqrt{2} w$ with

$$
w=\frac{\pi \rho_{0}-x^{11}}{V_{0}} 6 \pi \sqrt{2} l_{11}^{3}(k-12)
$$

where $x^{11}$ is a coordinate along the $S^{1} / \mathbb{Z}_{2}$ direction and $k$ is the number of fivebranes at the $x^{11}=0$ end of the interval. In these formulas, $\pi \rho_{0}$ is the size of the $S^{1} / \mathbb{Z}_{2}$ as measured with the coordinate $x^{11}$ and $V_{0}$ is the volume of the K3 as measured in the K3 metric $d K^{2}$. These along with $c$ are three of the moduli of the solution (the others being the other moduli of the K3 metric $d K^{2}$ ). The result (2.2) is valid in the regime $V_{0}>>l_{11}^{4}$ and $\rho_{0}>>V_{0}^{1 / 4}$.

Consider an M2-brane held at a point $y$ in the interval, and wrapped on a cycle of the $\mathrm{K} 3$ with area $A(y)=A_{0} y^{2 / 3}$. Because the interval is very large $\left(\rho_{0}>>l_{11}\right)$, the eleven-dimensional three-form gauge potential $C_{M N P}$ has a Kaluza-Klein excitation with a tunably small mass. This means that our wrapped M2-brane can be made stable to a good approximation by taking the interval large enough.

Its energy, taking into account the warping, is

$$
E=\sqrt{g_{00}} m_{0}=y^{-1 / 6}\left(y^{2 / 3} \frac{A_{0}}{l_{11}^{3}}\right)=y^{1 / 2} \frac{A_{0}}{l_{11}^{3}}
$$

where $m_{0}=y^{2 / 3} \frac{A_{0}}{l_{11}^{3}}$ is the proper energy of the wrapped M2-brane.

The extent of this wrapped brane along the $x_{\|}$directions is on the other hand given by $(1.2)$

$$
R=\frac{1}{\sqrt{a(y)}} r_{0}(y)
$$

in terms of a characteristic proper size $r_{0}$. We will presently argue that this characteristic size is in fact $l_{11}$. Taking this value, we obtain from (2.4)

$$
R=l_{11} y^{\frac{1}{6}}
$$

Combining (2.3) and (2.5), we obtain an energy-size relation

$$
E=T R^{3}
$$

where the effective tension $T$ is

$$
T=\frac{A_{0}}{l_{11}^{6}}
$$


It is tempting to infer a mass scale for excitations from this relation of the order $\tilde{\mu} \sim T^{1 / 4}$. However the scale of the tension is a priori not the only one in this problem; the kinetic energy of the elastic state will in general depend on further parameters. Therefore we will have to wait for the KK analysis of $\S 2.2$ to obtain a determination of the scale of excitations of this system.

Now let us fix $r_{0}$ for this example. We can do so in two different regimes that will be of interest. Firstly, consider the case where $A(y)>>l_{11}^{2}$ for all $y$ relevant to a given process. In this regime the supergravity solution for a wrapped membrane has a profile of characteristic size $l_{11}$ [9], plus small corrections down by powers of $l_{11}^{2} / A$. At the scale $l_{11}$, the supergravity/general relativity approximation breaks down and we expect new structure at this scale. We therefore expect this scale to be seen by appropriate $11 d$ supergravity modes in scattering processes, and we take it as a natural choice of the intrinsic proper thickness $r_{0}$ of the object.

Let us also consider the case of a regime of the K3 moduli space where $d K^{2}$ describes a K3 near an ALE singularity, so that there are 2-cycles in the K3 with area $A_{0}<<l_{11}^{2}$, and moreover consider a range of $y$ for which $A(y)<<l_{11}^{2}$. (We still take $V_{0}>>l_{11}^{4}$ to be able to continue to use the results (2.1) (2.2). Strictly speaking the supergravity analysis [1] does not apply if there are sub-Planckian defects in the manifold, but the warping is caused by excess fivebranes on one side of the interval scaling up the volume of the K3, which will presumably still happen in a regime where the K3 has localized ALE singularities. The analogous question has been studied in the Calabi-Yau threefold case in [10].) The UV cutoff of the gauge theory obtained near an ALE singularity (where the wrapped M2branes emerge as Higgsed gauge bosons of an ADE gauge theory) is $1 / l_{11}$. Therefore $l_{11}$ is the short-distance cutoff of the theory, plus possibly small corrections going like powers of $A / l_{11}^{2}$. This is also the scale of the gauge coupling of the nonrenormalizable gauge theory obtained at the ALE singularity. The Coulomb forces between the Higgsed gauge bosons will go like $1 / r^{4}$ for separations $r$ much greater than the cutoff $l_{11}$. The form factor for scattering of these states will then have a characteristic size of order $l_{11}$. So in this case also, we will take $l_{11}$ as the proper size $r_{0}$ of our M2-brane states.

Finally, for a collection of $N$ M2-branes, we will take $r_{0}$ to be $l_{11} N^{1 / 6}$, which is the characteristic scale appearing in the supergravity solution. In $\S 2.3$ we will study these regimes in more detail given the behavior of the KK wavefunctions we determine in the next subsection. 


\subsection{HW Kaluza-Klein Analysis}

We will now compute the spectrum of Kaluza-Klein modes of the wrapped M2-branes in the geometry (2.1). Let us consider for simplicity here the case of an M2-brane wrapped on a genus zero cycle of K3. For these the normal bundle is the line bundle $\mathcal{O}(-2)$ (which has no sections), so the M2-brane cannot move on the K3 without cost in energy. Here we will focus on the motion of the wrapped branes in the $y$ direction, which dominates over the motion in the K3 in appropriate regimes of moduli. Let us for simplicity also consider the scalar components of the wrapped M2-branes.

In order to calculate the spectrum of excitations of these wrapped M2-branes, we can use a Kaluza-Klein reduction of the action

$$
S_{H W}=\int d^{6} x_{\|} d y \sqrt{G}\left[-G^{M N} \partial_{M} \phi \partial_{N} \phi-m_{0}^{2}(y) \phi^{2}\right]
$$

Here the indices $M, N$ run over the seven dimensions of the warped interval, and $G_{M N}$ is given by the corresponding components of (2.1), so that $G_{\mu \nu}=\eta_{\mu \nu} y^{-1 / 3}$ and $G_{y y}=$ $y^{2 / 3} \frac{V_{0}^{2}}{l_{11}^{6}}$. There no explicit dependence in the action on the $y$-dependent K3 volume $V(y)=$ $V_{0} y^{4 / 3}$ since the curve is isolated; there is dependence on the cycle area $A(y)=A_{0} y^{2 / 3}$ through the mass term in (2.8).

The modes are given by solutions of the equation of motion

$$
\partial_{M}\left(\sqrt{G} G^{M N} \partial_{N} \phi\right)=\sqrt{G} m_{0}^{2}(y) \phi
$$

This becomes, upon plugging in our background,

$$
y^{-1 / 3} \partial_{x_{||}}^{2} \phi+\frac{l_{11}^{6}}{V_{0}^{2}} \partial_{y}\left(y^{-4 / 3} \partial_{y} \phi\right)=y^{2 / 3} \frac{A_{0}^{2}}{l_{11}^{6}} \phi
$$

Letting $\phi=e^{i k_{\|} x_{\| \mid}} \tilde{\phi}$ we have that $\partial_{x_{||}}^{2} \phi=-k_{\| \mid}^{2} \phi=\mu^{2} \phi$ where $\mu$ is the mass of the excitation in six dimensions.

Defining $\eta=y^{-2 / 3} \phi$, this becomes

$$
\eta^{\prime \prime}-\left[\frac{10}{9 y^{2}}+a y^{2}-b y\right] \eta=0
$$

where

$$
a=\frac{A_{0}^{2} V_{0}^{2}}{l_{11}^{12}} \quad b=\frac{\mu^{2} V_{0}^{2}}{l_{11}^{6}}
$$


This is related to a non-relativistic quantum mechanics problem with effective potential

$$
V(z)=a y^{2}-b y+\frac{10}{9 y^{2}} .
$$

We are interested in the discrete set of values of $\mu^{2}$ (which comes into the parameter $b$ (2.12)) for which this quantum mechanics problem has a state with zero energy eigenvalue.

For simplicity let us work in a regime where the last term in (2.13) can be dropped. The problem then reduces to a harmonic oscillator potential. We will be interested in determining the mass scale $\mu$ and the locations $y_{c}$ where the solutions $\eta$ are peaked. We will then check for self-consistency of this approximation.

In this regime, (2.11) can be rewritten

$$
-\eta^{\prime \prime}+a\left(y-\frac{b}{2 a}\right)^{2} \eta=\frac{b^{2}}{4 a} \eta
$$

The energy eigenvalues in the corresponding harmonic oscillator problem are given by

$$
E_{n}=\frac{b_{n}^{2}}{4 a}=\sqrt{a}\left(n+\frac{1}{2}\right)
$$

Using this and (2.12) we find a tower of masses

$$
\mu_{n}^{2}=\frac{2 A_{0}^{3 / 2}}{V_{0}^{1 / 2} l_{11}^{3}} \sqrt{\left(n+\frac{1}{2}\right)}=\frac{1}{\lambda_{C}^{2}}
$$

where we have indicated the Compton wavelength determined by this mass scale in the last step.

The nontrivial locations of the peaks of the wavefunction are at the length scale

$$
y_{n}=\frac{b}{a}=\frac{2 l_{11}^{3} \sqrt{n+\frac{1}{2}}}{A_{0}^{1 / 2} V_{0}^{1 / 2}}
$$

The values (2.17) are consistent with our assumption that the $1 / y^{2}$ term in (2.13) could be ignored relative to the linear and quadratic terms for large enough $\mu^{2}$.

From (2.17) and (2.5), we can determine the effective size of these excitations:

$$
R_{n} \sim y_{n}^{1 / 6} l_{11}=\mu_{n}^{1 / 3} \frac{l_{11}^{2}}{A_{0}^{1 / 3}}
$$

They satisfy a mass-size relation

$$
\mu_{n} \sim T R_{n}^{3}
$$


with a tension $T$ agreeing with that found in our analysis in $\S 2.1$.

Since these excitations have a nontrivial size $R_{n}$, the point-particle Kaluza-Klein analysis we have done breaks down at this scale, which is to say that the mode solutions of (2.9) apply only for momentum $k_{\|}$along the Poincare slices which is smaller than the scale $1 / R_{n}$.

Note that these states grow (in number) faster than for example Kaluza-Klein modes on a circle. Of course the states of a single elastic state grow much more slowly than those of say a perturbative string. In $\S 4.1$ we will discuss briefly possible bound states of these objects, whose density of states does appear to grow exponentially with some power of the energy.

\subsection{Size Scales and Regimes of Moduli}

In order for (2.2) to be reliable, we need $V_{0}>>l_{11}^{4}$. We can then consider different regimes of area $A(y)$ and M2-brane number $N$. Our arguments at the beginning of this section fixing $r_{0}=l_{11}$ involved assuming $A(y)$ to be either very large or very small relative to $l_{11}^{2}$ for the range of $y$ 's of interest. Let us check here that that can be arranged for our KK excitations (2.16) (2.17). At the typical $y$-values $y_{n}$, we have

$$
A\left(y_{n}\right)=\frac{2 A_{0}^{2 / 3} l_{11}^{2}\left(n+\frac{1}{2}\right)^{1 / 3}}{V_{0}^{1 / 3}}
$$

Suppose we wish to arrange for $A(y)>>l_{11}^{2}$. From $(2.20)$ this requires

$$
A_{0}^{2 / 3}\left(n+\frac{1}{2}\right)^{1 / 3}>>V_{0}^{1 / 3}
$$

In this regime, the membrane is wrapped on a very large 2-cycle, and is therefore heavy and behaves classically. Indeed, the condition (2.21) is the same as the condition that the Compton wavelength be much smaller than the object: $\lambda_{C}<<R_{n}$. When (2.21) is

satisfied for all $n$, the $\mathrm{K} 3$ is rather skew in shape, since in particular $\left(\frac{A_{0}}{l_{11}^{2}}\right)>>\left(\frac{V_{0}}{l_{11}^{4}}\right)^{1 / 2}$. In this regime the elastic states behave classically.

On the other hand, suppose we wish to consider the case where $A(y)<<l_{11}^{2}$. Then we need

$$
A_{0}^{2 / 3}\left(n+\frac{1}{2}\right)^{1 / 3}<<V_{0}^{1 / 3}
$$

For this regime, the Compton wavelength is large compared to the size scale $R_{n}$, and the objects behave very quantum mechanically. When the K3 is near an ALE singularity, we 
argued at the beginning of this section that the cutoff scale and gauge coupling scale $l_{11}$ determines the size of the object in this regime as well.

In both regimes we have studied here, for a small number $N$ of branes the size $R$ we have been discussing is determined by the warping from a fundamental proper size $r_{0}=l_{11}$. Although we expect structure at this scale as discussed above, this being the scale of the profile of the supergravity solutions for M-branes for example, we do not have direct control over the details of processes at this scale. 1 To study the elastic states' physics in a classical regime where we do have control, we can introduce a large number $N$ of wrapped M2-branes. Then as discussed above, $r_{0} \sim N^{1 / 6} l_{11}$. As in studies of black hole systems (11] etc.) the branes then become amenable to a classical analysis in certain regions of the solution [12].

Of course in adding branes to increase $N$, we increase both the mass and the size of the object as a function of $N$. Our point here is that in addition to that well-known effect, at fixed $N$ there is the effect we have identified here: the stretching of the object along $x_{\|}$ due to the warping as one increases its energy. Considering a fixed large $N$ allows us to study the classical physics of the elastic state effect in a regime where the physics at the scale of the object is under control.

We have thus obtained a consistent description of the wrapped M2-branes in the HW geometry which shows that they grow in size as a function of their energy. In this sense they behave like extended objects. We will begin a study in $\S 4$ of the question of the extent to which these objects and their bound states might behave as familiar extended objects such as branes.

\section{The Type I' Case}

In this section we will do a similar analysis of NS5-branes in the type I' geometry derived in [2]. We will work this case out a little more schematically than the last one, since the procedure is hopefully clear, but it is worth exhibiting a second example of the basic effect under consideration.

2 Perhaps a more tractable generalization would be a case involving a (possibly wrapped) perturbative string. In such a case one could calculate reliably scattering processes at the relevant size scale $\left(l_{s}\right)$. 
By some rescalings of the moduli and coordinates defined in [2] we can write the string-frame metric and dilaton schematically in the form

$$
\begin{gathered}
d s_{\text {string }}^{2}=\frac{\gamma^{\frac{5}{3}}}{(\beta+w)^{\frac{1}{3}}}\left(d x_{\|}^{2}+l_{s}^{2} d w^{2}\right) \\
e^{\phi}=\frac{1}{[\gamma(\beta+w)]^{\frac{5}{6}}}
\end{gathered}
$$

where $\beta$ and $\gamma$ are some combinations of the dilaton and radial modulus of the type I' theory. Here $x_{\|}$denotes the coordinates along the $9 d$ Poincare slices in this geometry.

Let us compactify 5 of the spatial $x_{||}$dimensions on a $T^{5}$. For simplicity let us take the simple square shape

$$
x_{\|}^{5, \ldots, 9} \cong x_{\|}^{5, \ldots, 9}+R_{0}
$$

The thickness of $N$ NS5-branes in the remaining $x_{\|}$directions can be determined in a large $N$ limit from the supergravity solution. This gives 13]

$$
r_{0} \sim l_{s} N^{1 / 2}
$$

We can determine the energy-size relation from the warped metric and dilaton as follows. Let us work in terms of a coordinate $y \equiv w+\beta$. Taking into account the variation of the volume of the $T^{5}$ and the variation of the dilaton as a function of $y$, the energy is

$$
E=\sqrt{g_{00}} m_{0}=\frac{R_{0}^{5}}{l_{s}^{6}} \gamma^{20 / 3} y^{2 / 3}
$$

The size is

$$
R=\frac{y^{1 / 6}}{\gamma^{5 / 6}} l_{s} N^{1 / 2}
$$

Putting these together leads to a growth in size with energy:

$$
E=T R^{4}
$$

with effective tension

$$
T=\frac{R_{0}^{5} \gamma^{10}}{l_{s}^{10} N^{2}}
$$

The Kaluza-Klein analysis in this case can be done similarly to that for the HoravaWitten case. 


\section{Disussion: Bound States, Scattering, and Other Issues}

\subsection{Remarks on Bound States and Branes}

Though our elastic states have a growth in size with energy somewhat reminiscent of branes, they each individually only have a Kaluza-Klein theory's worth of states. However bound states of elastic states might exist (establishing or ruling out this possibility would require a careful analysis such as was done for D0-branes [14]). If so, these could have a much faster (exponential) growth in number with energy.

In the HW case, the wrapped M2-branes experience gravitational attraction and gauge $\left(C_{M N P}\right)$ repulsion. Because the $S^{1} / \mathbb{Z}_{2}$ direction is compact, the relevant modes of $C_{M N P}$ are slightly massive, as discussed in $\S 2$. Therefore at very long distances the different elastic states attract each other. This suggests bound states might be possible, though an analysis of the short-distance structure is required to establish (or rule out) this possibility.

Consider a bound collection of bound states of $N_{n}$ elastic states at level $n$ in the spectrum (2.16) derived in $\S 2$. An upper bound on the energy of such a state (in that it does not take into account the binding energy) is

$$
\mu_{t o t} \sim \sum_{n} N_{n} \mu_{n}=\sum_{n} N_{n}\left(n+\frac{1}{2}\right)^{1 / 4} \frac{\sqrt{2} A_{0}^{3 / 4}}{V_{0}^{1 / 4} l_{11}^{3 / 2}}
$$

This spectrum grows exponentially.

If bound states do exist here, it is tempting to speculate that a large number of elastic states could mock up an effective brane (perhaps in a somewhat analogous way to the way D0-branes mock up higher branes in matrix theory [15]). It is further tempting to speculate that this approach can then provide a new way to quantize effective branes and study their interactions in some nontrivial regime.

\subsection{Remarks on Scattering Amplitudes}

Given the growth in size with energy of the objects we have been studying, it is interesting to consider where this effect would arise in scattering amplitudes. Contact interactions between large and small elastic states (which are particles at different points $y_{1}, y_{2}$ in the interval) are suppressed by the separation between $y_{1}$ and $y_{2}$. As in the case of objects going by each other in the AdS bulk [16], in the $d$-dimensional description our objects appear to pass right through each other.3

3 One possibility is that the large objects form a ring instead of a filled-in ball in the $x_{\|}$ dimensions as in some states in AdS [3] [5]. 
However if we consider the electromagnetic and gravitational interactions of our objects, their size is evident at tree level. In particular, the thickness of the object leads to a form factor with characteristic scale $R$ in gauge and gravity-mediated scattering processes.

It will be interesting to study the effects of elastic states and bound states of elastic states in loops. For example it will be interesting to calculate the contribution these objects and their bound states make to the vacuum energy. For this we need to know the spectrum of bound states as well as a controlled prescription for describing their interactions. It may be very useful to consider an analogous case where the elastic states originate as strings (with a warping which amplifies their $l_{s}$-scale size).

\subsection{Discussion}

There are many issues to explore further with these states (and their many cousins in other warped compactifications). Generalizations which could be important for a potential brane picture include cases where the wrapped branes oscillate in more than one direction of a warped compactification. As discussed above, a case which would give more tractable calculations would be one in which the basic object is a perturbative string whose mass and size get warped by the compactification so as to give an energy-size relation of the kind we have studied here.

One question involves the relevance of AdS/CFT ideas and results to more generic warped compactifications. In some ways the elastic states we have discussed here are a generalization of the baryon states identified in AdS/CFT duals and their generalizations [8]. The growth in size of states with energy is characteristic of gravity in various highenergy regimes, but can also occur for some collective excitations in ordinary quantum field theory (and exponential growth of the density of states can also occur there in certain regimes [17]). It would be interesting to classify the behavior of states at high energy in QFT vs gravity in this regard, and in particular to understand if the class of states studied

here is characteristic of gravity or could occur in a theory with a completely quantum field-theoretic dual.

Another interesting application of warped geometries is potentially to cosmology (obtained in appropriate cases by viewing the direction along which the warping appears as time $t$ ). In such a situation, the wrapped branes can become zero-action instantons at a singularity occuring at $t=0$ in the cosmology. It would be interesting to understand whether the corresponding instanton sum accounts for (and is related to a resolution of) the initial singularity in this sort of setup. 
Particularly in the context of gravity, the effects of nonlocality are potentially important for many problems (see e.g. [18] for a recent application). It would be interesting to understand what role if any these extended states play in the nonlocality of gravity in warped compactifications.

\section{Acknowledgements}

I would like to thank A. Adams, O. Aharony, N. Arkani-Hamed, T. Banks, M. Berkooz, S. Kachru, A. Lawrence, J. Maldacena, M. Peskin, S. Shenker, and A. Strominger for useful discussions. This work is supported by a DOE OJI grant, by the A.P. Sloan Foundation, and by the DOE under contract DE-AC03-76SF00515. 


\section{References}

[1] E. Witten, "Strong Coupling Expansion Of Calabi-Yau Compactification," Nucl. Phys. B471, 135 (1996) hep-th/9602070.

[2] J. Polchinski and E. Witten, "Evidence for Heterotic-Type I String Duality," Nucl. Phys. B460 (1996) 525, hep-th/9510169.

[3] J. Polchinski, L. Susskind and N. Toumbas, "Negative energy, superluminosity and holography," Phys. Rev. D60, 084006 (1999) hep-th/9903228.

[4] N. Kaloper, E. Silverstein and L. Susskind, "Gauge symmetry and localized gravity in M theory," hep-th/0006192.

[5] S. Giddings, talk at Aspen 2000 String Workshop.

[6] J. McGreevy, L. Susskind and N. Toumbas, "Invasion of the giant gravitons from Anti de Sitter space," JHEP 0006, 008 (2000), hep-th/0003075.

[7] L. Susskind and E. Witten, "The holographic bound in anti-de Sitter space," hepth/9805114; A. W. Peet and J. Polchinski, "UV/IR relations in AdS dynamics," Phys. Rev. D59, 065011 (1999) hep-th/9809022; V. Balasubramanian, P. Kraus, A. Lawrence and S. P. Trivedi, "Holographic probes of anti-de Sitter space-times," Phys. Rev. D59, 104021 (1999) hep-th/9808017.

[8] E. Witten, "Bound States and Branes in Anti-de-Sitter Space", JHEP 9807, 006 (1998) hep-th/9805112.

[9] M. J. Duff and K. S. Stelle, "Multi-membrane solutions of D = 11 supergravity," Phys. Lett. B253, 113 (1991).

[10] A. Lukas, B. A. Ovrut, K. S. Stelle and D. Waldram, "Heterotic M-theory in five dimensions," Nucl. Phys. B552, 246 (1999) hep-th/9806051; A. Lukas, B. A. Ovrut, K. S. Stelle and D. Waldram, "The universe as a domain wall," Phys. Rev. D59, 086001 (1999) hep-th/9803235.

[11] A. Strominger and C. Vafa, "Microscopic Origin of the Bekenstein-Hawking Entropy," Phys. Lett. B379, 99 (1996) hep-th/9601029.

[12] N. Itzhaki, J. M. Maldacena, J. Sonnenschein and S. Yankielowicz, "Supergravity and the large N limit of theories with sixteen supercharges," Phys. Rev. D58, 046004 (1998) hep-th/9802042.

[13] C. G. Callan, J. A. Harvey and A. Strominger, "Supersymmetric string solitons," hep-th/9112030.

[14] S. Sethi and M. Stern, "D-brane bound states redux," Commun. Math. Phys. 194, 675 (1998) hep-th/9705046.

[15] T. Banks, W. Fischler, S. H. Shenker and L. Susskind, "M theory as a matrix model: A conjecture," Phys. Rev. D55, 5112 (1997) hep-th/9610043; T. Banks, N. Seiberg and S. Shenker, "Branes from matrices," Nucl. Phys. B490, 91 (1997) hep-th/9612157. 
[16] L. Susskind, private communication; S. Giddings, E. Katz, and L. Randall "Linearized gravity in brane backgrounds," JHEP 0003, 023 (2000) hep-th/0002091.

[17] B. Kol, "Thermal monopoles," JHEP 0007, 026 (2000) hep-th/9812021.

[18] T. Banks, "Cosmological breaking of supersymmetry or little Lambda goes back to the future II," hep-th/0007146. 